\title{
حول استبعاد جرائم الإرهاب الاولي من الاختصاص الموضوعي للمحكمة الجنائية الدولية من الائية
}

On the material incompetence of the International Criminal Court on the crimes of international terrorism

تاريخ الاستلام : 2019/06/11 ؛ تاريخ القبول : 20191/06/25

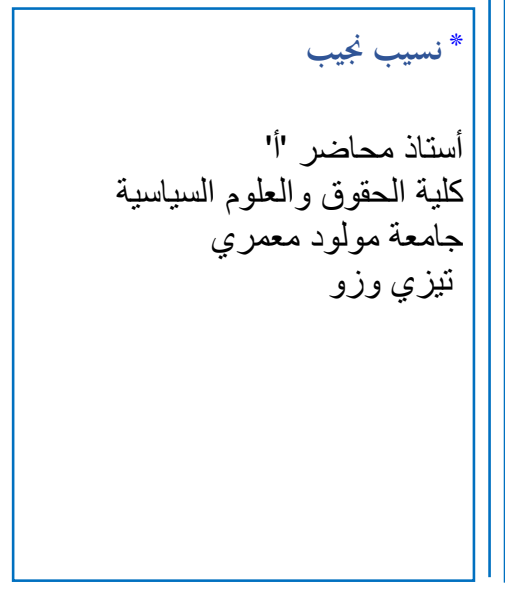

\section{Abstract}

The problem with the ICC is the problem of its matrial jurisdiction, which at present covers only classic international crimes, war crimes, crimes against humanity and genocide.The ICC Statue does not contain any provision on terrorist acts, all attempts to do so during negociations at the failed rome conference.

In general, when drafting the Roma Statue, the definition of crimes falling within the jurisdiction of the ICC was a thomy issue. Opponents of the inclusion of international terrorism in the court's jurisdiction have expressed legal and political reservations.

Keywords: crimes of international terrorism, the international criminal court, material jurisdiction of the international criminal court international criminal court, legal causes, political causes.

\section{Résumé}

Le problème majeur en ce qui concerne la CPI est le problème de sa compétence matérielle, qui à l'heure actuelle ne recouvre que les crimes internationaux classiques, crimes de guerre, crimes contre l'humanité et génocide. Le statut de la CPI ne contient en effet pas de dispositions concernant les actes terroristes, toutes les tentatives faites dans ce sens durant les négociations a la conférence de Rome ayant échoué .

En général, lors de la rédaction du Statut de Rom, la définition des crimes relevant de la compétence de CPI était une question épineuse. Des opposants à l'inclusion du terrorisme international dans la compétence de la Cour ont exprimé des réserves juridiques et politiques.

Mots clés: crimes de terrorisme international, la cour pénale internationale, la compétence matérielle de la cour pénale internationale, les causes juridiques, les causes politiques.

* Corresponding author, e-mail: nacibnadjib@gmail.com 
أضحى الإرهاب في الوقت الر اهن ظاهرة واسعة الانتشار تبعث على القلق،

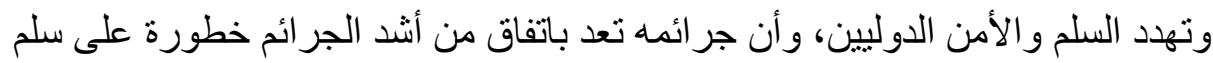
ورفاه البثرية.

ولما كان الأمر كذللك، فإن كافة الظروف ومعطيات الواقع أضحت تدعو وتلح

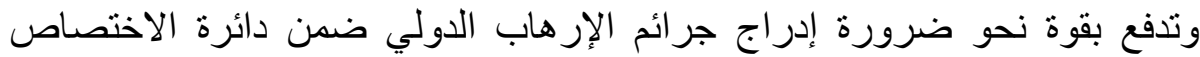

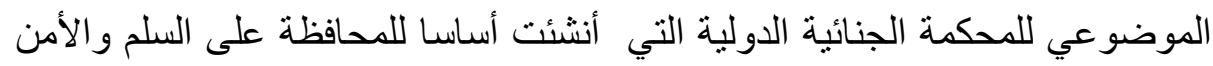

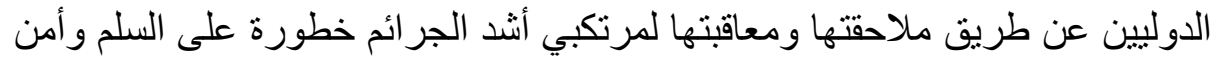

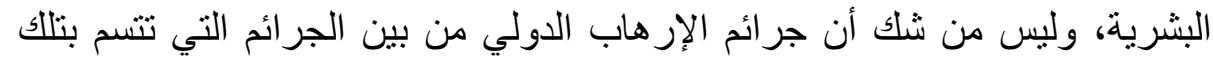

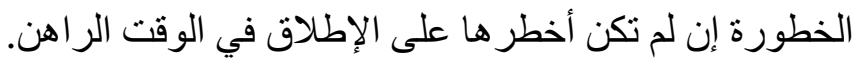

غير أن إدراج جرائم الإرهاب الدولي ضمن الاختصاص الموضوعي

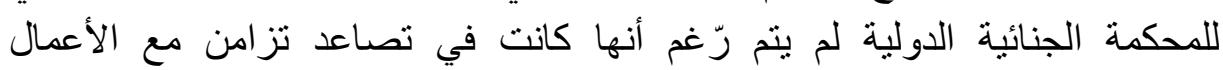

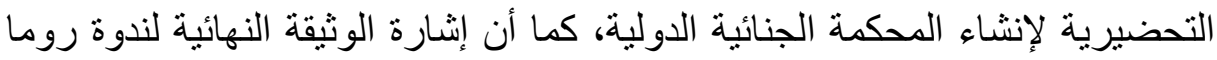

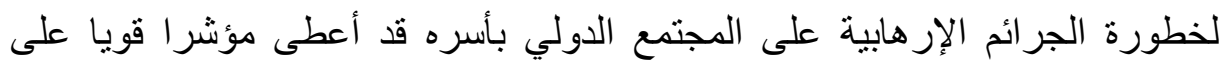
إدراج الإرهاب الدولي ضمن الجرائم التي تختص المحكمة الجنائية الدولية بتتبعها لإنها ومكافحتها إلاّ أن هذا الأمر لم يتم(1).

وبصفة عامة، فتحديد ماهية الجرائم الداخلة في اختصاص المحكمة الجنائية

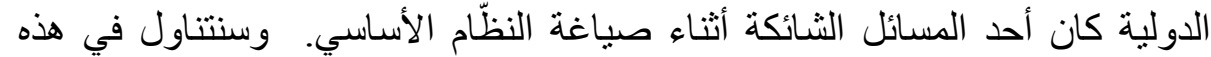
الدراسة أهم الأسباب التي أدت إلى عدم إدراج جرائم الإرهاب الداء الدولي ضمن الدمن

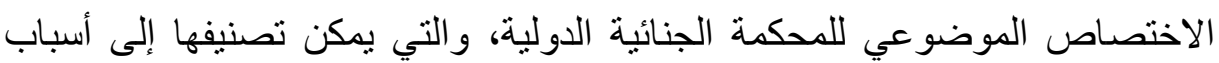
قانونية (المطلب الأول) ، و أخرى سياسية (المطلب الثاني).

$$
\text { المطلب الأول: الأسباب القانونية }
$$

بلغت أهمية مكافحة الإرهاب الدولي حدا كبير ا دفع الدول والمنظّمات الدولية

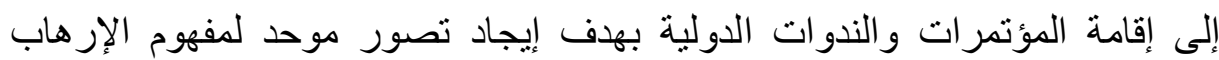
يتسنى بموجبه للمجتمع الدولي وضع إستر اتيجية فعالة لمكافحته ومعاقبة مرتكبيه.

ومن بين أهم هذه المؤتمرات مؤتمر الأمم المتحدة الدبلوماسي للمفوضين

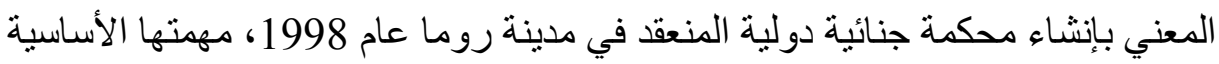

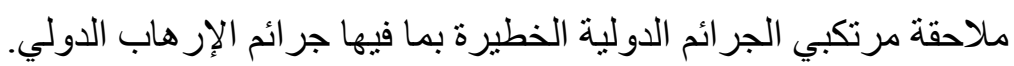

غير أن من يتمعن النظر في الأحكام الواردة في نظام روما الأساسي بعد

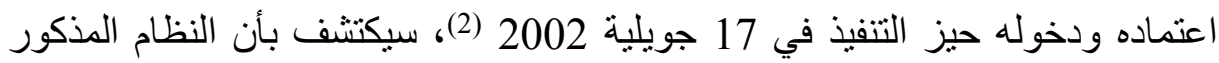
جاء خاليا من أي حكم يتعلق بتعريف الإرهاب وبالتالي افتقاره للنص على مكافحة وقمع 
وقد تمحورت أهم التحفظات القانونية التي أبدتهاوفود الدول المشاركة في ندوة

روما لعدم إدراج الإرهاب في الاختصاص الموضوعي للمحكمة الجنائية الدولية في

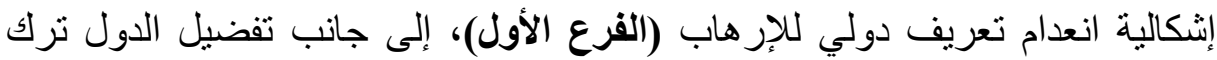

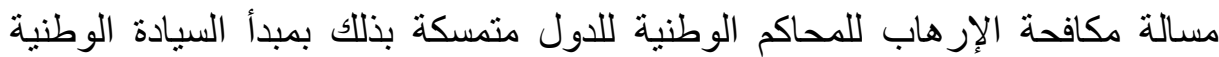
المطلقة (الفرع الثاني). الفرع الأول: انعدام تعريف شامل ومتفق عليه للإرهاب

دخلت فكرة الإرهاب عالم الفكر القانوني لأول مرة في المؤتمر الدولي الأول

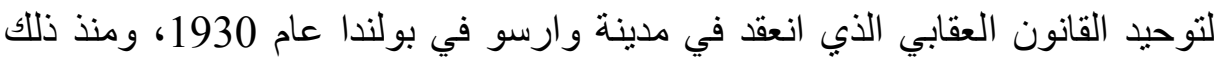

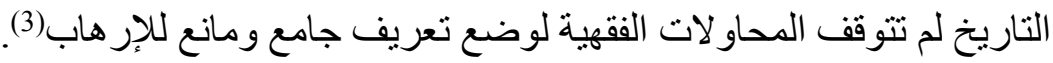

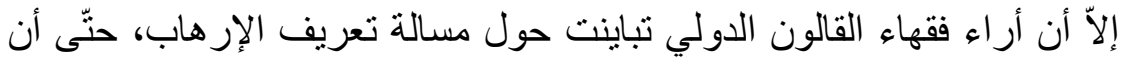

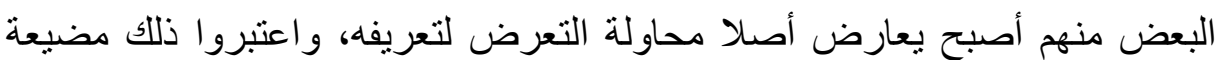

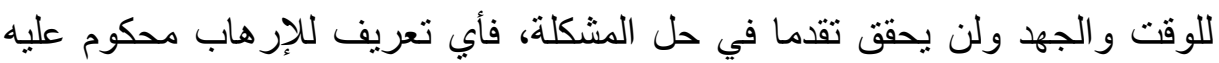

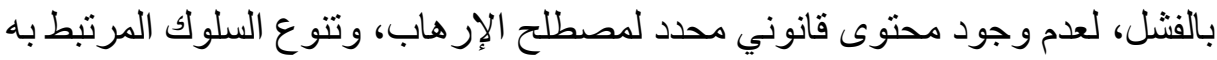

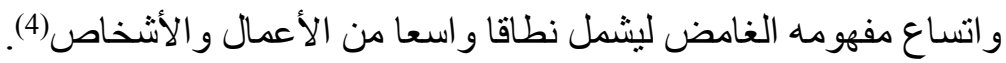

وقد قامت لجنة القانون الدولي و بطلب من الجمعية العامة للأمم المتحدة

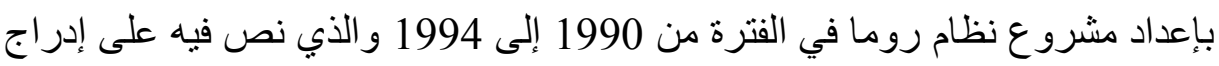
جريمة الإر هاب في الاختصاص الموضوعي للمحكمة الجنائية الدولية، وتمت صياغة ذللك النص على النحو التالي: \الأغراض هذا النظام الأسناسي، تعني جرائم الإرهاب:

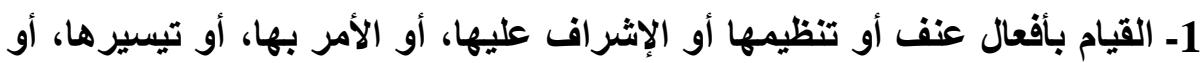

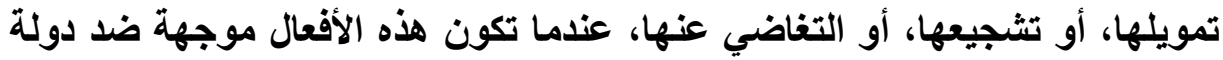

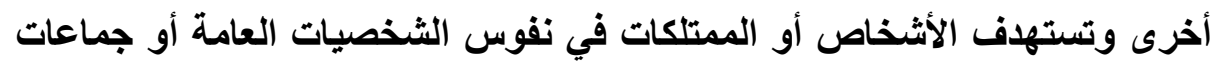

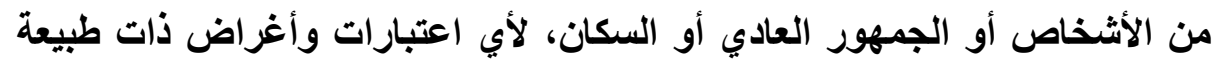

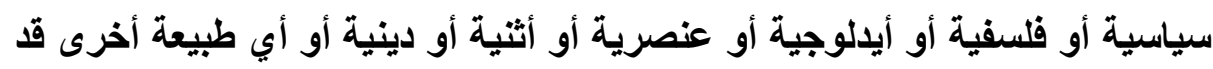
يحتج بها لتبريرها.

2- جريمة بموجب الاتفاقيات التالية:

أ ـاتفاقية قمع الأعمال غير المشروعة ضد سلامة الطيران المدني (اتفاقية مونتريال).

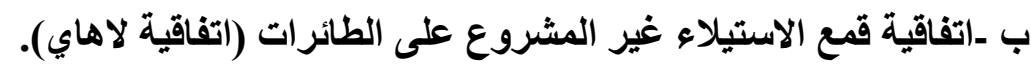

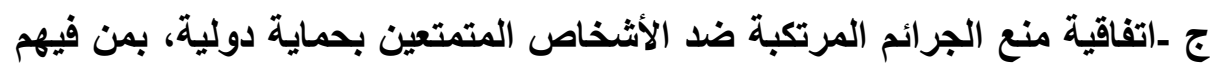
الموظقون الدبلوماسيون والمعاقبة عليها.

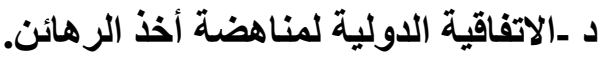

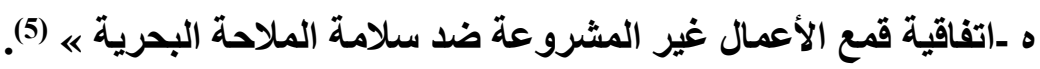




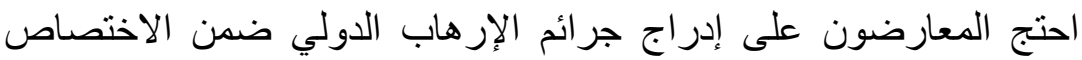

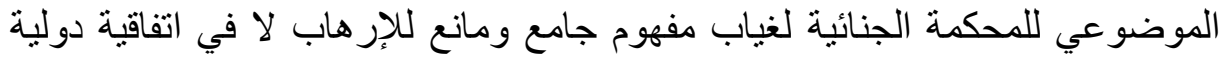
موحدة، ولا في القانون الدولي العام، إذ بعد الإرهاب ـرّغم انتشاره الواسع وآثاره

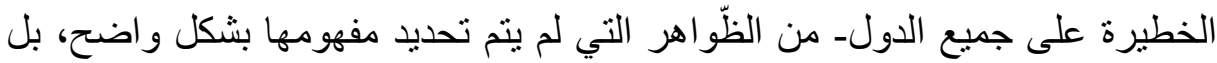
وتوجد بشأنها تناقضات واضحة في التعامل معها والتصدي لها بإجراءات تشريعية و وعقابية مختلفة(6).

و عليه أصبحت صلاحية المحكمة الجنائية الدولية بالنظر في جرائم الإرهاب

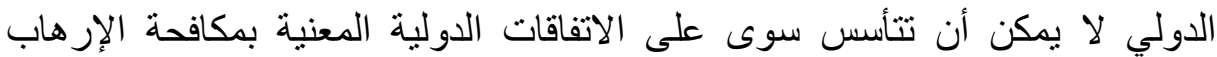

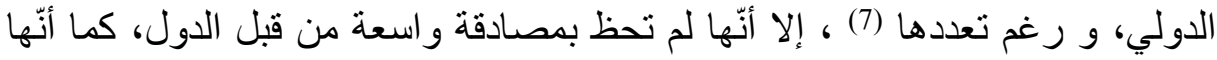
اتفاقات قطاعية تختص بمكافحة جر ائم إر هابية محددة، فهناك احتمال أن تكون الجمات الجريمة

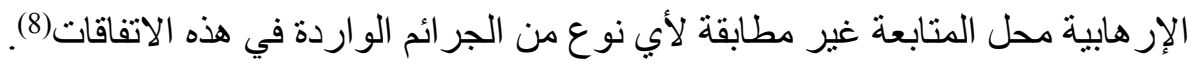

كما اعتبر بعض الفقهاء والدارسين لموضوعي الإرهاب و المحكمة الجنائية

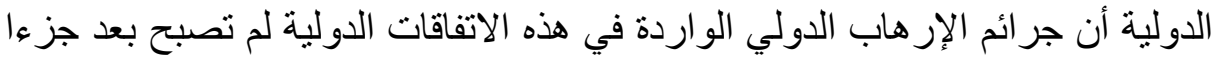
من القانون العرفي الدولي مثل غير ها من الجر ائم الأساسية(9).

فيرى البعض أنّ جر ائم الإر هاب و إنّ كانت تستحق القمع والإدانة إلاّ أنها لا

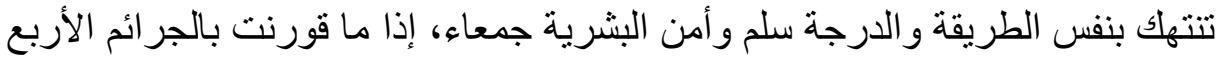

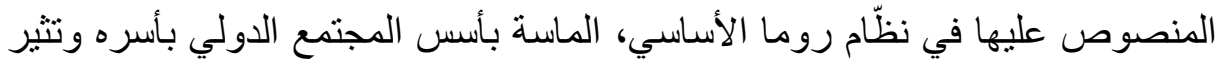
ضمير الإنسانية والتي تعتبر في الممّارسة الدولية والاجتهاد القضائي الدولي بمثابة الأبية

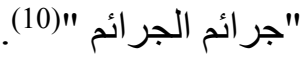

وكان من رأي المؤيدين لإدر اج جر ائم الإر هاب ضمن اختصاص المحكمة، أن

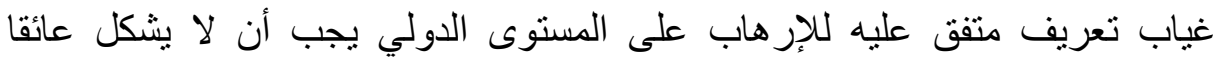

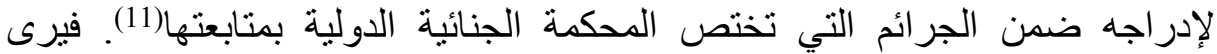

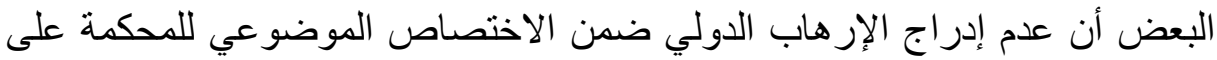

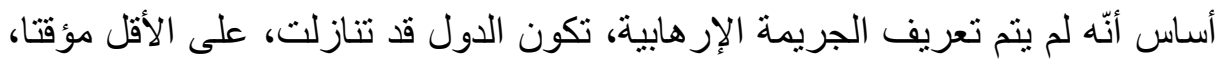
لإنشاء استجابة جنائية عالمية لمكافحة جر ائم الإر هاب(12).

\section{الفرع الثاني: ترك مسالة مكافحة الإرهاب للمحاكم الوطنية للدول}

رأت بعض الدول أن متابعة مرتكبي جرائم الإرهاب الدولي سيكون أكثر فعالية على المستوى الوطني (13)، فمن الأحسن ترك الاختصاص بها للمحاكم الوطنية في إطار الآليات التقليدية للتعاون القضائي الدولي، إذ أن إثقال كاهل المحكمة بمتابعة الإنة

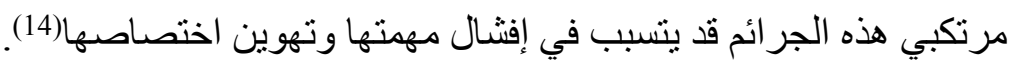
ورّد المؤيدون لإدراج جرائم الإر هاب ضمن اختصاص المحكمة على هذه 
الحجة بقولهم أن منح الاختصاص للهيئات القضائية الداخلية للاول بالنظر في جرائم الإرهاب الدولي سيؤدي حتما إلى انحلال قمع هذه الجرائم تحت وطأة تعدد الأحكام

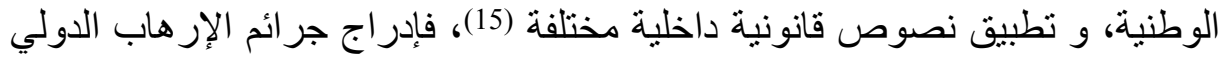

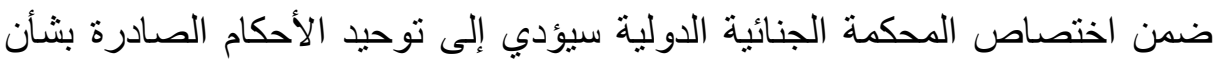
هذه الجر ائم، ويسمح للدول بتفادي إشكالات التنازع القضائي في متابعة مرتكبيها (16) . كما أن إدراج جرائم الإرهاب الدولي ضمن اختصاص المحكمة الجنائية

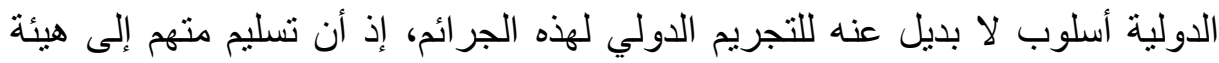
قضائية دولية لا يؤثر بنسبة كبيرة على سيادة الدولة المطلوب منها ذلابه اللك مقارنة بنسليمه إلى الدولة الطالبة للتسليم(17).

\section{المطلب الثاني: الأسباب السياسية}

إن عدم إدر اج جر ائم الإرهاب الدولي ضمن الاختصاص الموضوعي للمحكمة الجنائية لا يرجع فقط للأسباب القانونية التي سبق ذكر ها بل توجد أسباب سياسية أبدتها وفود الدول المشاركة في ندوة روما والتي حالت دون إمكانية إسهام المحكمة الجنائية

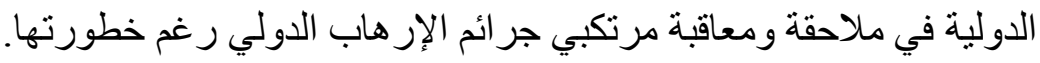

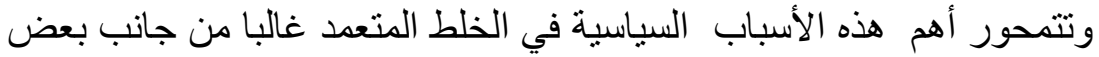

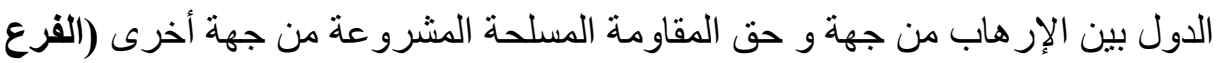
الأول)، إلى جانب إبداء الدول لتحفظات متعلقة بمخافة تسييس حمل الإدكمة الجنائية

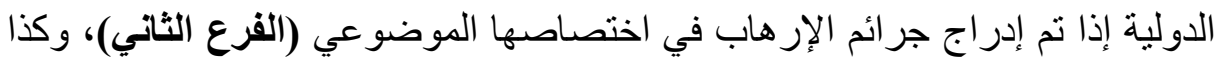
سعي بعض الدول الكبرى إلى وضع العراقيل لأداء هذه المحكمة مهامها (الفرع الدهائ)

\section{الفرع الأول: الخلط بين الإرهاب وحق المقاومة المسلحة المشروعة}

يكمن السبب الرئيسي في تباين مواقف الدول حول إدراج جرائم الإرهاب

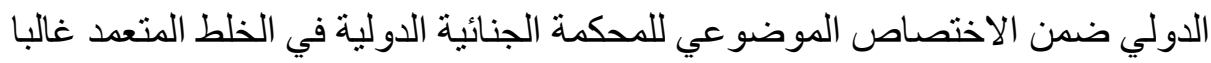

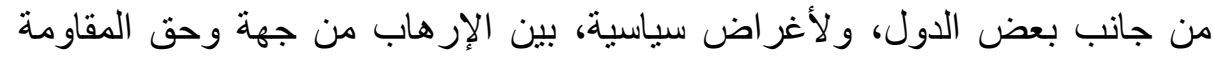

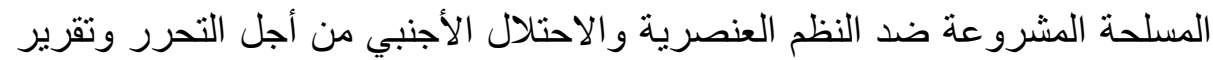
المصير من جهة أخرى(18).

إذ أن من أصعب المشاكل القانونية التي واجهت الدارسين لوضع تعريف للإر هاب الدولي هي مسألة التفرقة بين أعمال الإرهاب والأعمال التئ التي تقوم بها حركات

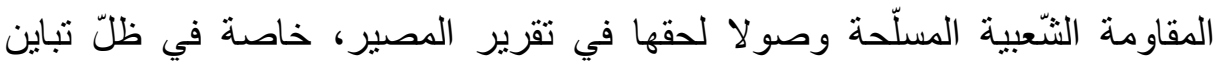

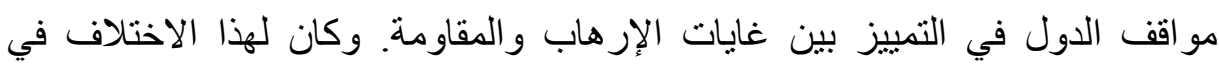

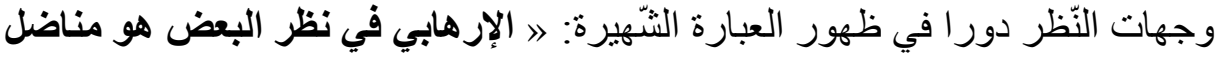
في سبيل الحريّة في نظر البعض الآخره(19) . 


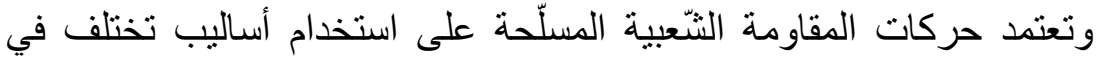

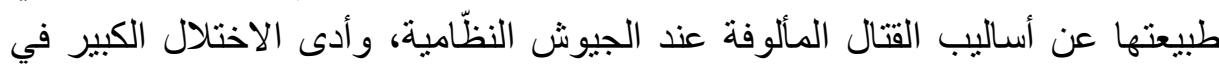

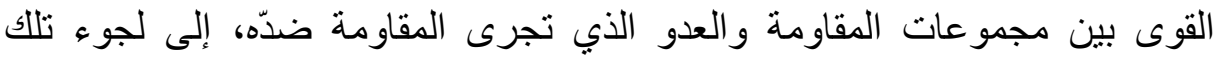

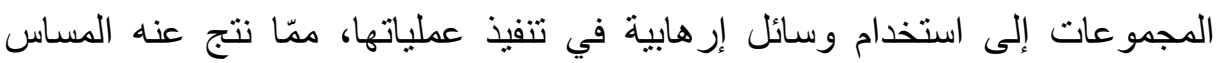
بسلامة المدنيين في الكثير من الأحوال(20.

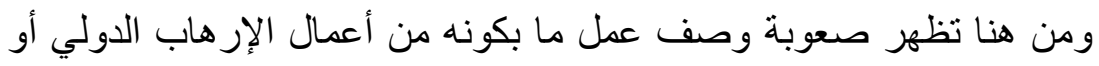

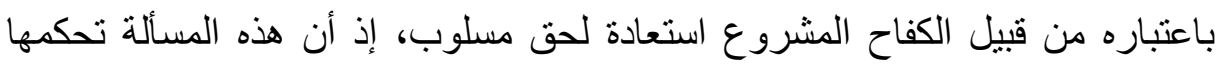

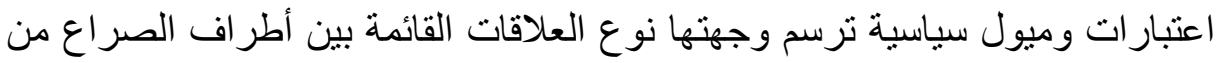

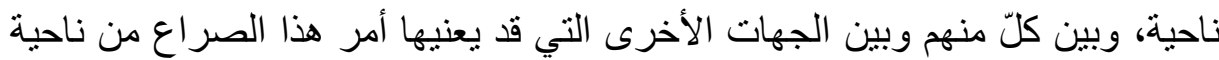

أخرى(21).

وبعد سقوط الثنائية القطبية وسيادة قيم الغرب، أدين الكفاح المسلّح، واعتبر من من مانِ

أعمال الإر هاب، ما يدل على أن مفهوم المقاومة و الإر هاب مفهومان نسبيان لا يرتبطان

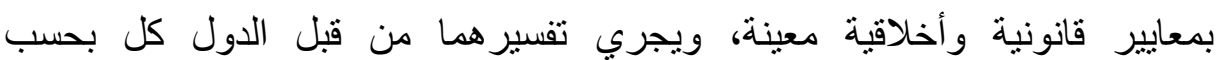
مصالحهائ(22).

وقد ساهمت هذه الاعتبارات السياسية وتباين مواقف الدول حول الحدود

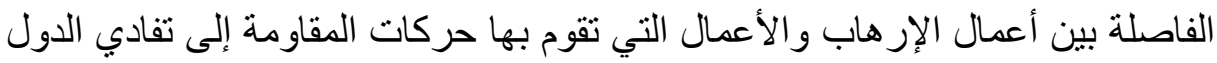

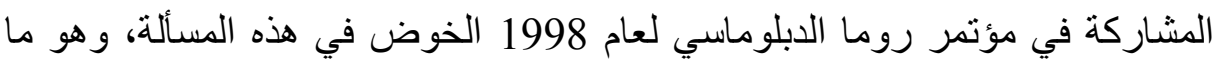

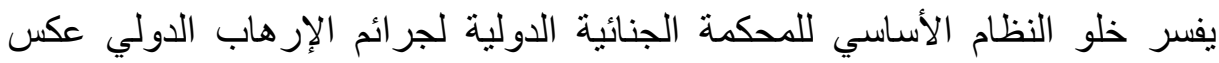
مشرو عه.

\section{الفرع الثاني : الخوف من تسييس المحكمة الجنائية الدولية}

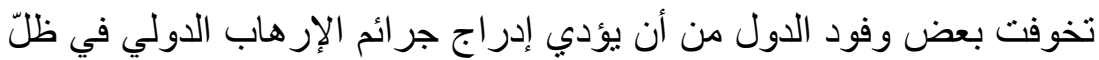

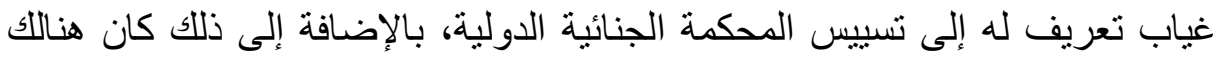
عدم اتفاق بشأن أنشطة حركات التحرر وتمييز ها عن الأعمال الإر هابية(23). وخشية أن يفثل المؤتمر إذا أعيد النقاش حول طبيعة جرائم الإرهاب، قرر

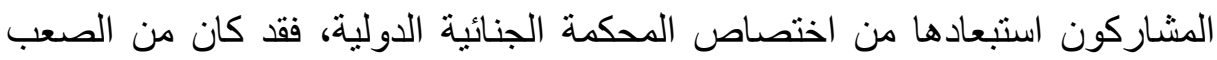
التوصل إلى اتفاق بشأنها و لا سيما أن فترة انعقاده كانت قصيرة التهادئن نسبيا (24) .

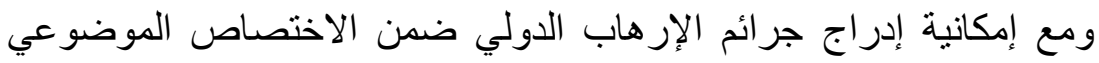
للمحكمة الجنائية الدولية في المستقبل إلاّ أن هناك بعض الته العقبات العبات السياسية التي تحول

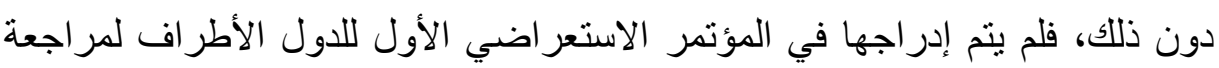

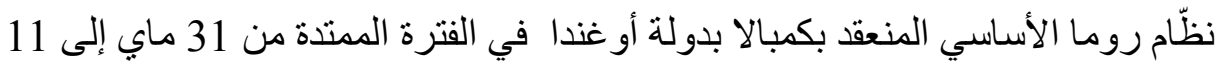

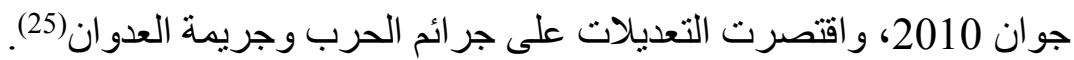


كما أن تردد العديد من الدول المصادقة على النظّام الأساسي للمحكمة لخشيتها

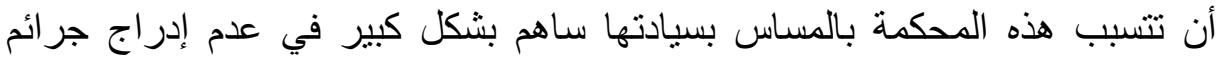

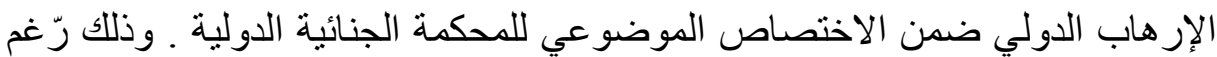
من أن العديد من هذه الدول عانت وما زالت تعاني من ويلات الإرهاب ( كالهند،

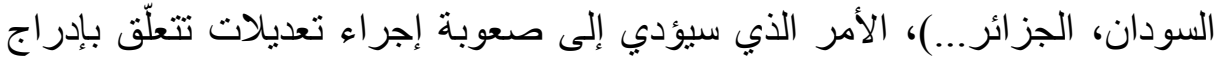
جر ائم الإر هاب وغير ها من الجرائم الدولية ذات الخطورة ضمن اختصاصاتها، نظر الترات

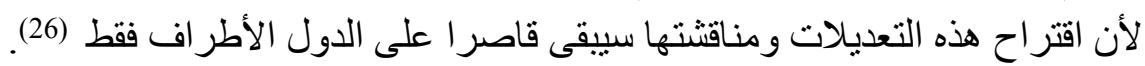

الفرع الثالث: قيام بعض الدول الكبرى بوضع العراقيل لأداء المحكمة الجنائية

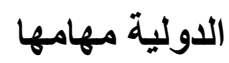

قامت بعض الدول الكبرى بوضع العر اقيل لأداء هذه المحكمة مهامها، ومنها

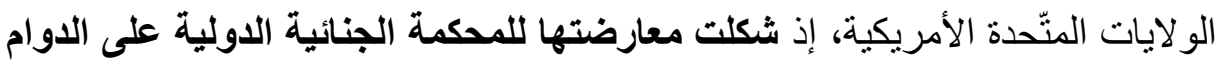

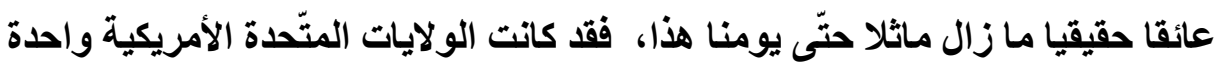

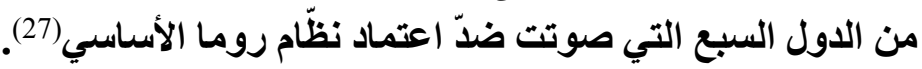

وترجع هذه المعارضة إلى سبيين رئيسيين : يتمثل الأول في رفض المجتمع

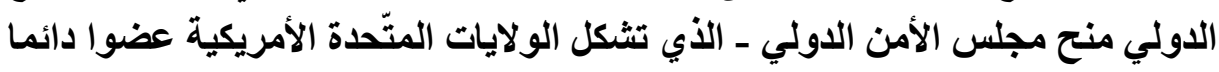

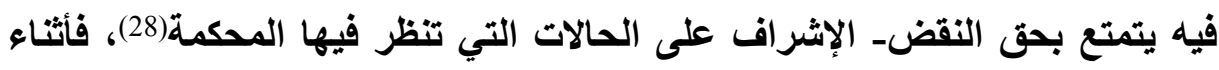

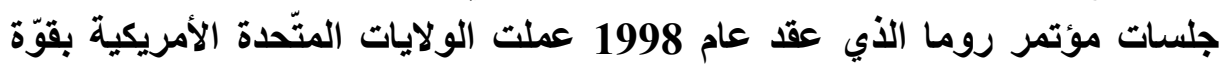

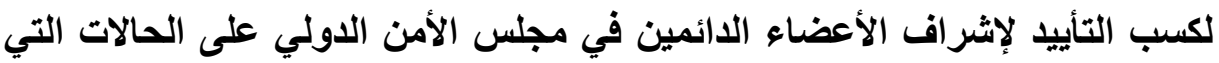

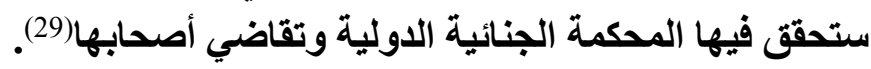

لكن المؤتمر أفضى إلى نتيجة مغايرة من خلال نظّام روما الأساسي الذي مدي

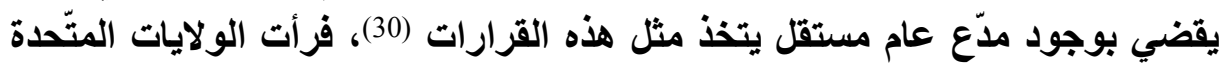

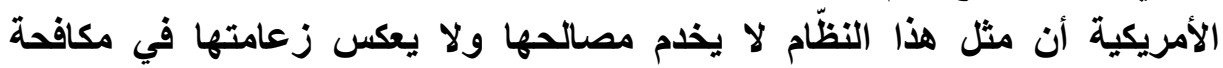

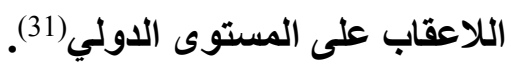

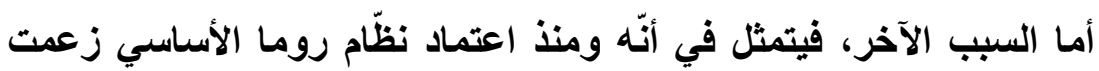

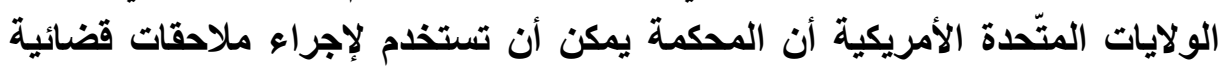
نابعة من أهداف سياسية ضدّ رعاياهات(32).

ورّ غم من توقيع الو لايات المتّحدة الأمريكية على نظّام روما في 31 ديسمبر

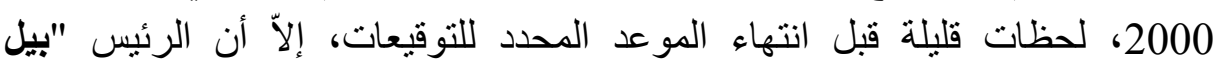

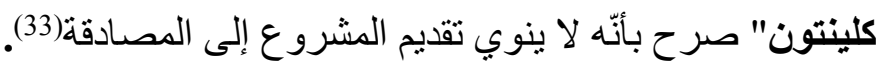

وفي إطار الحرب على الإرهاب التي أعلنتها الولايات المتّحدة الأمريكية

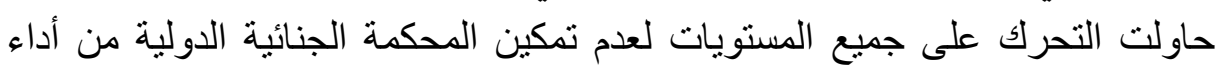




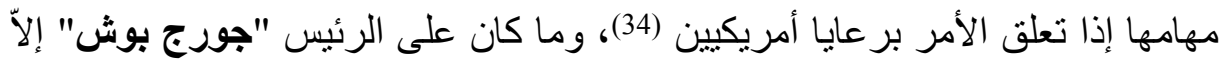

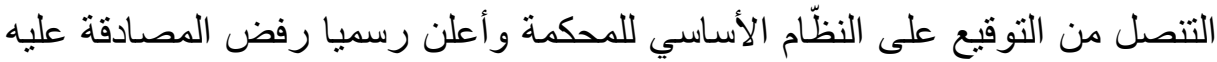

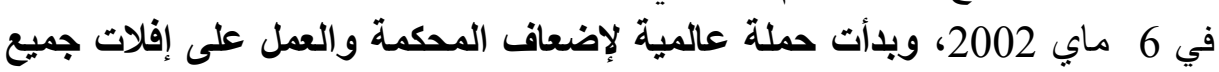
المواطنين الأمريكيين من العقاب الصادر بموجب الولاية القضائية للمحكمة(35).

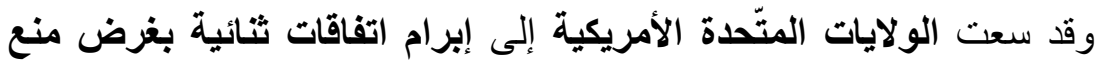

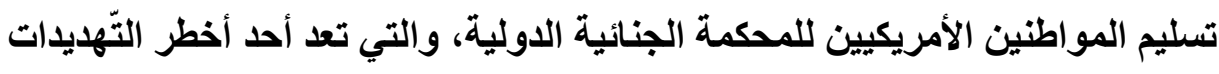

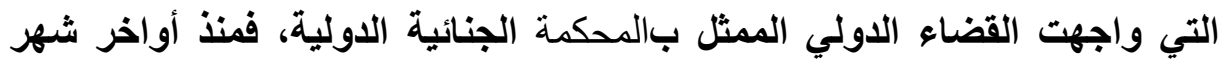

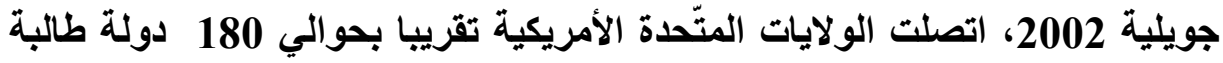

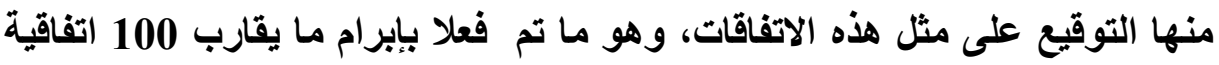

من خلال در استتا لأهم الأسباب التي حالت دون إدراج جر ائم الإرهاب الدولي الإبي

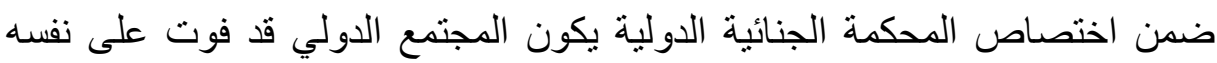

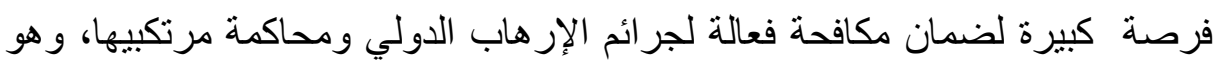
الموقف الذي أبدته العديد من الدول في ختام مؤتمر روما.

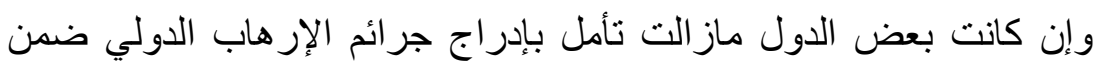

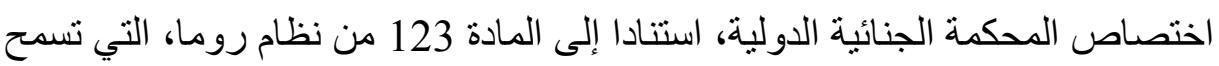

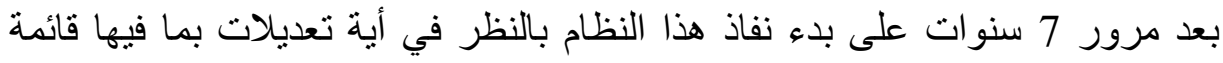

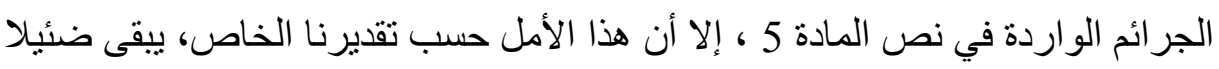

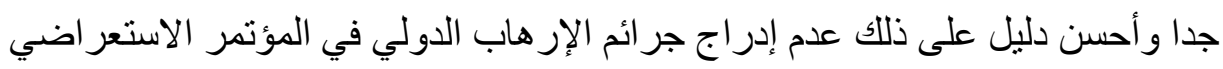

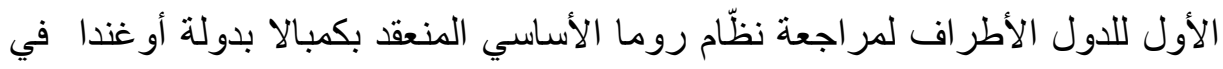

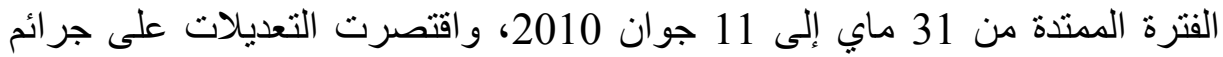
الحرب وجريمة العدوان. 
(1)- Sandra SZUREK, Le jugement des auteurs d'actes de terrorisme : Quels tribunaux après le 11 septembre ? in, Le droit international face au terrorisme après le 11 septembre, Ouvrage collectif, Karine BANNELIER, Théodore CHRISTAKIS, Olivier CORTEN, Barbara DELCOURT (S/D), Pedone, Paris , 2002, p.311; Anatole COLLINET MAKOSSO, Le terrorisme, de l'immunité à l'incrimination: Etude juridique d'une violence à réprimer par l'action combinée de la Cour pénale internationale et la Cour internationale de justice, Thèse de doctorat en droit privé, Université Panthéon-Assas Paris II, 2010, p. 517.

(2) - دخل نظّام روما الأساسي حيز التنفيذ في17 جويلية 2002 و اكتفت الجزائر بالتوقيع عليه في 2000/12/28.

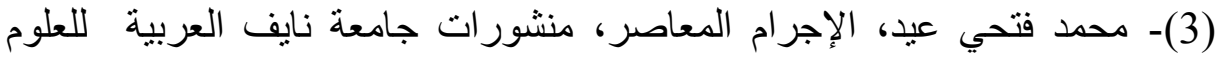

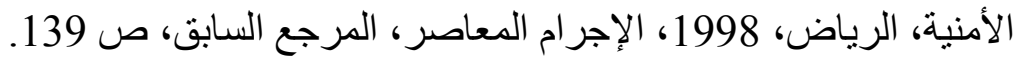

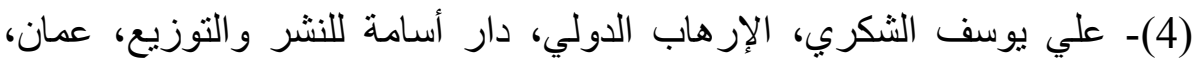

الأردن، 2008، ص 22.

- Marie Elisabeth CARTIER, Le terrorisme dans le nouveau code pénal français, RSC, juillet septembre 2000, p. 228.

(5) - نقلا عن: محمد هاثم ماقورا، أهم أسباب عدم اختصاص المحكمة الجنائية الدولية بجر ائم الإرهاب الدولي بحث مقدم في المؤتمر الدولي لجامعة الحسين بن طلال

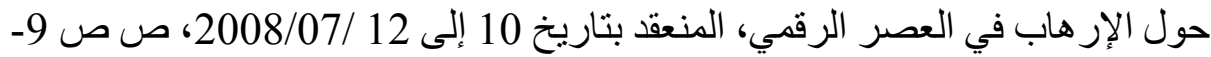

(6)- محمد عبد الرحيم سلطان العلماء، معوقات تطوير أنظمة العدالة الجنائية في

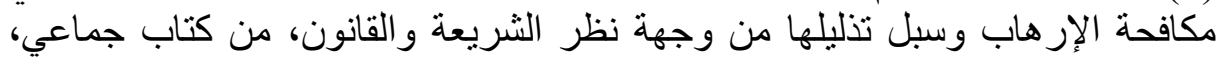

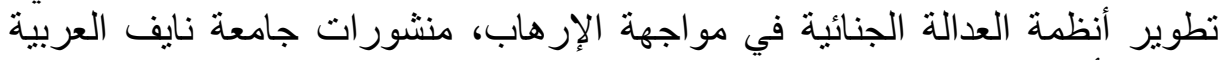

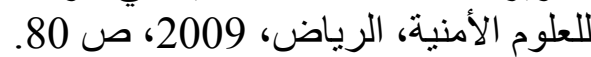
(7) - تنمثل هذه الاتفاقات فيما يلي:

ـ اتفاقية طوكيو لـنع الجرائم وبعض لاتئ الأفعال الأخرى المرتكبة على منن الطائرات، لسنة 1963. ـ اتفاقية مكافحة الاستيلاء غير المشروع على الطائر ات، لسنة 1970.

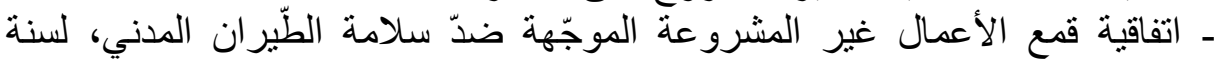
.1971 ـ اتفاقية منع الجرائم المرتكبة ضدّ الأشخاص المتمتعين بحماية دولية، بمن فيهر الموظفون الدبلوماسيون، و المعاقبة عليها، لسنة 1973. ـ ـ اتفاقية مناهضة احتجاز الر هائن، لسنة 1979.

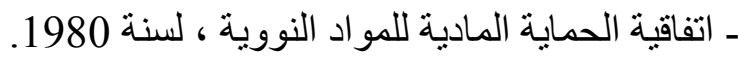

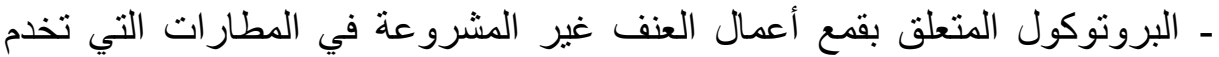

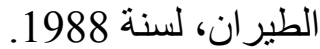
ـ اتفاقية قمع الأعمال غير المشروعة الموجّهة ضدّ سلامة الملاحة البحرية، لسنة 
ـ البروتوكول المتعلق بقع الأعمال غير المشروعة الموجّهة ضدّ سلامة المنشآت

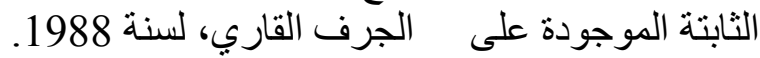

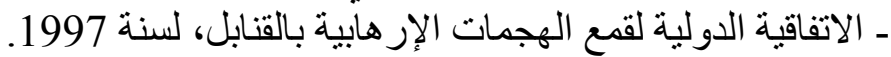

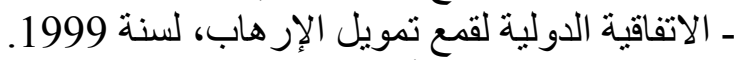

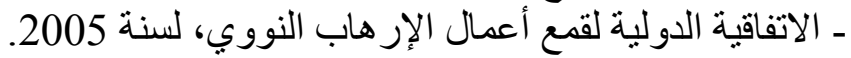

(8)- Sandra SZUREK, Op.Cit., p. 316; Anatole COLLINET MAKOSSO, Op.Cit., p. 517.

(9)- Hala EL AMINE, Pourquoi la Cour pénale internationale n'est - elle pas compétente en matière de terrorisme international ? in, Terrorisme et droit international, RCADI, 2008, p. 252 ; Philippe KIRSCH, Terrorisme, crimes contre 1'humanité et Cour pénale internationale, in, S.O.S ATTENTATS, Livre noir

- Terrorisme et responsabilité pénale internationale - Paris, 2002, p. 114.

(10)- Sandra SZUREK, Op.Cit., p. 317.

(11)- Ghislaine DOUCET, Terrorisme : Recherche de définition ou dérive liberticide ? in, Terrorisme, victimes et responsabilité pénale internationale, DOUCET Ghislaine (S/D), Calmann-Levy, Paris, 2003, p. 400.

- يرى البعض أن مكافحة الإرهاب الدولي بشكل فعال بتطلب إنشاء محكمة جنائية

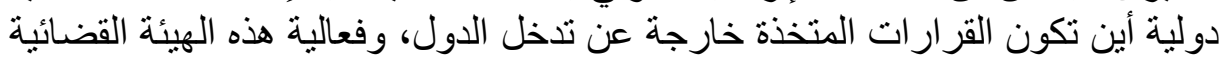

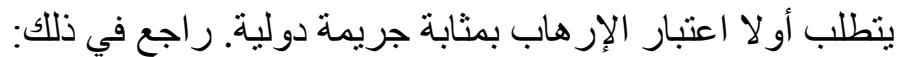

- Reynald OTTENHOF, Approche criminologique et victimologique $\mathrm{du}$ terrorisme, in, Terrorisme, Victimes, et responsabilité pénale internationale, Op.Cit., p. 499.

(12)- Ghislaine DOUCET, Terrorisme : Définition, juridiction pénale internationale et victimes, RIDP, Vol. 76, 2005, p. 252.

(13)- نصر الدين بوسماحة، حقوق ضحايا الجرائم الدولية على ضو ألح أحكام القانون

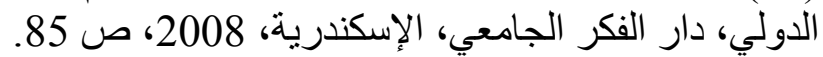
(14)- Philippe KIRSCH, Op.Cit., p. 114 ; Sandra SZUREK, Op.Cit., p.317.

(15)- Sandra SZUREK, Op.Cit., p. 312.

(16)- Hala EL AMINE, Op.Cit., pp. 247- 249.

(17)- Ibid, p. 249.

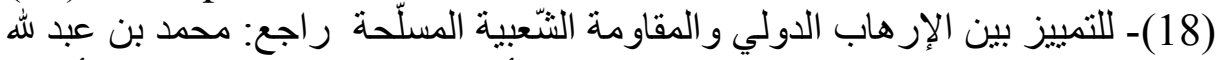

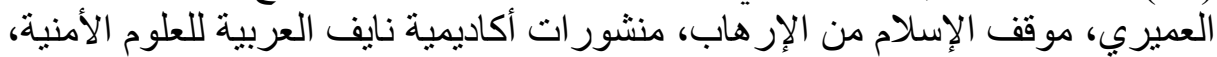

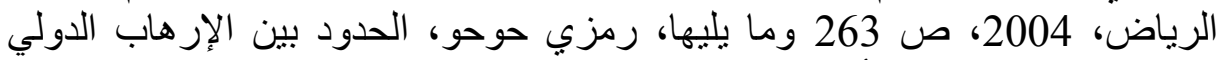

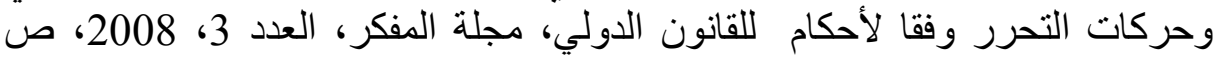
161

(19)- محمد إبراهيم الحلوة، الإرهاب الدولي في ماهيته وأسبابه وسبل مكافحته،

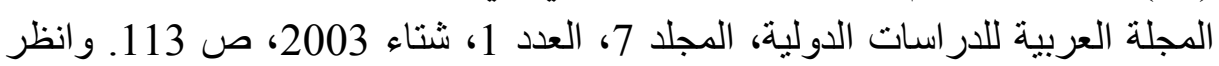


- Isabelle SOMMIER, Le terrorisme, Dominos Flammarion, France, 2000, p. 85.

(20)- سامي جاد عبد الرحمن و اصل، إرهاب الدولة، منشأة المعارف، الإسكندرية، ص ص 243. (20) صنامي

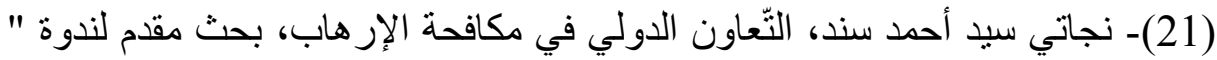

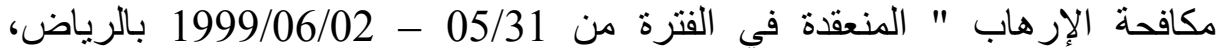
منشور ات أكاديمية نايف العربية للعلوم الأمنية، الرياض، الاضه

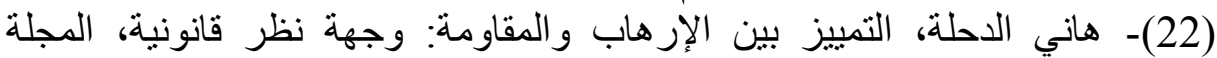

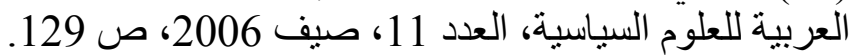
(23)- Hala EL AMINE, Op.Cit., p. 253; Philippe KIRSCH , Op.Cit., pp. 114 - 115.

(24)- علاء الدين راشد، المشكلة في تعريف الإرهاب، دار النهضة العربية، القاهرة،

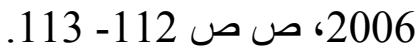
(25)- للمزيد من التفاصيل حول هذه التعديلات راجع نتائج المؤتمر الاستعر اضي لنظّام روما الأساسي على الموقع: http://www.icc-cpi.int/iccdocs/asp_docs/ASP9/OR/RC-11-

ARA.pdf

(26)- سامي جاد عبد الرحمن واصل، المرجع السابق، ص ص 501- 502.

(27) - Stéphanie MAUPAS, L'essentiel de la justice pénale internationale, Gualino Editeur, Paris, 2007, p. 137.

(28)- عادل حمزة عثمان، المحكمة الجنائية الدولية بين الثّرعية الدولية والهيمنة

الأمريكية، مجلة الكوفة للعلوم القانونية و السياسية، العدد 7، نوفمبر النية 2010، ص 71.

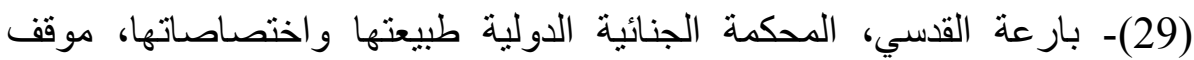

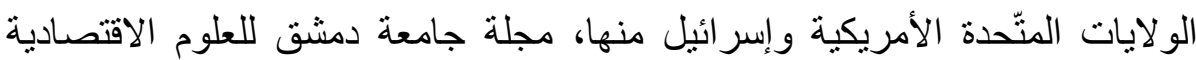

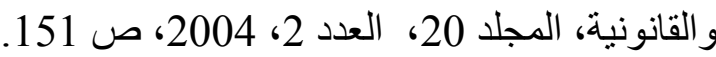
(30) - Frédérique COULEE, Sur un Etat tiers bien peu discret : Les États-Unis confrontés au statut de la Cour pénale internationale, AFDI, Vol. 49, 2003, pp. 39 - 40.

(31)- في الو اقع أن الو لايات المتّحدة الأمريكية أيدت دائما إنشاء محكمة جنائية دولية،

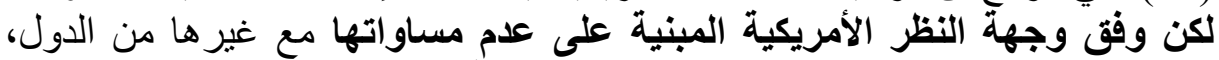

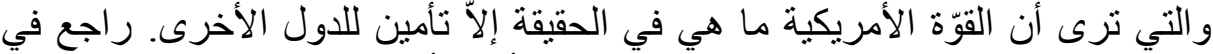

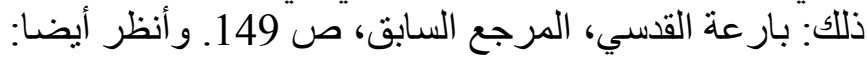

- Federica DAINOTTI, La Cour pénale internationale est une réalité, Diplôme des hautes études européennes et internationales, Institut européen des hautes études internationales 2005/2006, p p. $74-75$.

(32)- Federica DAINOTTI, Op.Cit., p. 76.

(33)- Anatole COLLINET MAKOSSO, Op.Cit., pp. 520 - 521 ; Frédérique COULEE, Op.Cit., p. 43.

- > بتوقيعنا على المعاهدة لم نتخل عن مخاوفنا بشأن الثغرات الموجودة فيها، خاصة 
أنّه لاى تثكل المحكمة ستمارس سلطاتها لا على مواطني الدول التي صادقت على التى التها

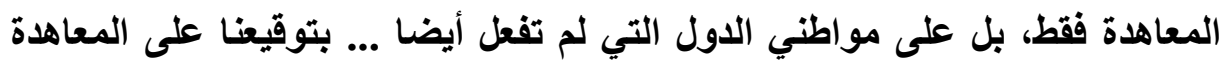

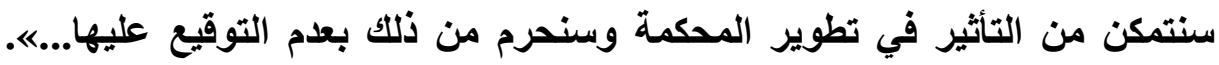

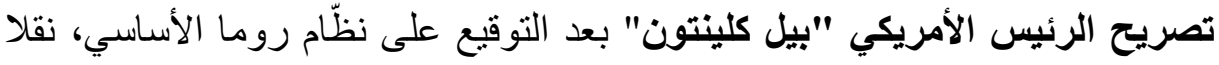

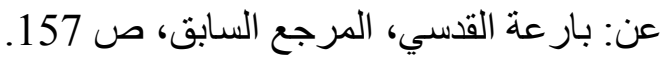
(34)- Jaques FORMERAND, La pratique américaine du multilatéralisme, le syndrome de la mare nostrum, AFRI, Vol. 4, 2003, p. 487.

(35)- Federica DAINOTTI, Op.Cit., p. 75; Frédérique COULEE, Op.Cit., p. 44 ; Stéphanie MAUPAS, Op.Cit., p. 138.

(36)- Federica DAINOTTI, Op.Cit., p. 80 ; Frédérique COULEE, Op.Cit., p. 58. 